\title{
A deep learning approach to diagnose atelectasis and attic retraction pocket with otoscopic images
}

Junbo Zeng ${ }^{1}$, wenting Deng ${ }^{2}$, Jingang $\mathrm{Yu}^{3}$, Lichao Xiao ${ }^{3}$, Suijun Chen ${ }^{1}$, Xueyuan Zhang ${ }^{4}$, Linqi Zeng ${ }^{5}$, Donglang Chen ${ }^{5}$, Peng Li ${ }^{6}$, Yubin Chen ${ }^{6}$, Hongzheng Zhang ${ }^{7}$, Fan Shu ${ }^{8}$, Jinliang $\mathrm{Gao}^{9}$, Minjian $\mathrm{Wu}^{1}$, Yuejia $\mathrm{Su}^{10}$, Yuanqing $\mathrm{Li}^{3}$, Yuexin $\mathrm{Cai}^{11}$, and Yiqing Zheng ${ }^{12}$

${ }^{1}$ Sun Yat-sen Memorial Hospital, Sun Yat-sen University

${ }^{2}$ Sun Yat-Sen University 2nd Affiliated Hospital

${ }^{3}$ South China University of Technology

${ }^{4}$ Sun Yat-sen Memorial Hospital of Sun Yat-sen University

${ }^{5} 4$. Zhongshan School of Medicine, Sun Yat-sen University

${ }^{6}$ the Third Affiliated Hospital of Sun Yat-Sen University

${ }^{7}$ Southern Medical University

${ }^{8}$ Nanfang Hospital, South Medical University

${ }^{9}$ Shenzhen Baoan Women's and Children's Hospital

${ }^{10}$ Sun Yat-Sen Memorial Hospital

${ }^{11}$ Sun Yat-sen Memorial Hospital, Sun Yat-sen University

${ }^{12}$ Sun yatsen university

January 17, 2022

\begin{abstract}
Background: Atelectasis and attic retraction pocket are two common tympanic membranes changes. However, general practitioners, pediatricians and otolaryngologists showed low diagnostic accuracy for these ear diseases. Therefore, there is a need to develop a deep learning model to detect atelectasis and attic retraction pocket automatically. Method: 6393 OME otoscopic images from 3 centers were used to develop and validate a deep learning model to detect atelectasis and attic retraction pocket. 3 -fold random cross validation was adopted to divided dataset into training set and validation set. A team of otologists were assigned to diagnose and label. Receiver operating characteristic (ROC) curve, 3-fold average classification accuracy, sensitivity and specificity were used to assess the performance of deep learning model. Class Activation Mapping (CAM) was applied to show the discriminative region in the otoscopic images. Result: Among all the otoscopic images, 3564 (55.74\%) images were identified with attic retraction pocket, and 2460 (38.48\%) images were identified with atelectasis. The automatically diagnostic model of attic retraction pocket and atelectasis achieved 3-fold cross validation accuracy of $89 \%$ and $79 \%$, AUC of 0.89 and 0.87 , sensitivity of 0.93 and 0.71 , and specificity of 0.62 and 0.84 respectively. Bigger and deeper atelectasis and attic retraction pocket showed more weight with red color in the heat map of CAM. Conclusion: Deep learning algorithm could be used to identify atelectasis and attic retraction pocket, which could be used as a tool to assist general practitioners, pediatricians and otolaryngologists. Key words: deep learning, otoscopic images, atelectasis, attic retraction pocket
\end{abstract}

\section{A deep learning approach to diagnose atelectasis and attic retraction pocket with otoscopic} images

Abstract 
Background: Atelectasis and attic retraction pocket are two common tympanic membranes changes. However, general practitioners, pediatricians and otolaryngologists showed low diagnostic accuracy for these ear diseases. Therefore, there is a need to develop a deep learning model to detect atelectasis and attic retraction pocket automatically.

Method: 6393 OME otoscopic images from 3 centers were used to develop and validate a deep learning model to detect atelectasis and attic retraction pocket. 3-fold random cross validation was adopted to divided dataset into training set and validation set. A team of otologists were assigned to diagnose and label. Receiver operating characteristic (ROC) curve, 3-fold average classification accuracy, sensitivity and specificity were used to assess the performance of deep learning model. Class Activation Mapping (CAM) was applied to show the discriminative region in the otoscopic images.

Result: Among all the otoscopic images, 3564 (55.74\%) images were identified with attic retraction pocket, and $2460(38.48 \%)$ images were identified with atelectasis. The automatically diagnostic model of attic retraction pocket and atelectasis achieved 3-fold cross validation accuracy of $89 \%$ and $79 \%$, AUC of 0.89 and 0.87 , sensitivity of 0.93 and 0.71 , and specificity of 0.62 and 0.84 respectively. Bigger and deeper atelectasis and attic retraction pocket showed more weight with red color in the heat map of CAM.

Conclusion: Deep learning algorithm could be used to identify atelectasis and attic retraction pocket, which could be used as a tool to assist general practitioners, pediatricians and otolaryngologists.

\section{Key Points}

- Atelectasis and attic retraction pocket are two common tympanic membrane changes.

- Otoscope is widely accepted for diagnosing and managing OME, atelectasis and attic retraction pocket.

- General practitioners, pediatricians and otolaryngologists showed low diagnostic accuracy for ear diseases.

- We developed a deep learning model to diagnose atelectasis and attic retraction pocket using otoscopic images and assess the performance of deep learning model.

- It may be used to improve the procedure of OME diagnosis and management, such as saving time and improving diagnostic accuracy.

\section{INTRODUCTION}

Atelectasis and attic retraction pocket occur because of tympanic membranes architectural deformity and bad ventilation, then tympanic membranes collapse toward to the tympanic cavity. Tympanic membrane retraction is the most common change of tympanic membrane in pediatric otitis media with effusion (OME) patients ${ }^{1}$. Atelectasis and attic retraction pocket also could be sequela of OME, and it's more frequently in surgery cases ${ }^{2,3}$. Patients with mild atelectasis or attic retraction pocket may have no symptoms, however, tiny attic retraction pocket may conceal attic cholesteatoma ${ }^{4}$. Severe atelectasis and attic retraction pocket may cause erosion of ossicular chain, outer wall of tympanic cavity and hearing loss. Moreover, whether there is atelectasis or attic retraction pocket is important part in the OME diagnosis procedure ${ }^{5}$. Cholesteatoma and adhesive otitis media are common severe sequela of atelectasis and attic retraction pocket ${ }^{6}$. Although surgery serves as an effective method to treat severe atelectasis and attic retraction pocket, but surgery is associated with economic burden, and surgery risk, such as sensorineural hearing loss, facial palsy. And some patients may cease to retract and returned to normal condition, so prophylactic surgery would not be recommended ${ }^{7}$. However, early diagnosis with appropriate follow up is a reasonable policy to manage atelectasis and attic retraction pocket, irrespective of surgery or not ${ }^{8,9}$.

Otoscope is widely accepted for diagnosing and managing OME, atelectasis and attic retraction pocket ${ }^{5,10}$. And many types of smartphone adaptable otoscopes can be used to acquire tympanic membranes images by no-specialty or no-clinicians ${ }^{11-13}$. However, Diagnosis of ear disease only with manual examination shows low accuracy, which may lead to improper referral, delayed or improper treatment and pointless follow-up.

The progressive use of telemedicine and artificial medicine in the otologic setting may gradually change the procedure of disease management. Wu et. al ${ }^{14}$ developed a deep learning model to diagnosis pediatric otitis 
media using otoscopic images and tested in a smartphone-enabled otoscope set. Shie et. al ${ }^{15}$ extracted color, geometric and texture features to develop a classification system for differentiating most type of otitis media, achieving an accuracy of $88.06 \%$ in 865 otoscopic images. Cha et. al ${ }^{16}$ developed a deep learning model to detect 6 common ear diseases acquiring an accuracy of $93.67 \%$. All the previous artificial intelligence studies didn't classify retraction of tympanic membranes as atelectasis and attic retraction pocket.

The purpose of this study was to develop a deep learning model to diagnose atelectasis and attic retraction pocket using otoscopic images and assess the performance of deep learning model. It may be used to improve the procedure of OME diagnosis and management, such as saving time and improving diagnostic accuracy.

\section{METHOD}

\section{Participant selection and otoscopic images acquisition}

Otoscopic images from inpatients and outpatients were collected retrospectively from 3 hospitals between year 2015 to 2019. Otoscopic images were taken with $4 \mathrm{~mm}$ (KARL STORZ, Germany) or $2.7 \mathrm{~mm}$ (TIAN SONG, China) 0-degree otoscope by otolaryngologists. OME cases were confirmed with criteria of clinical guideline $^{5}$, including disease history, medical examination and auditory test. 1 to 3 best quality otoscopic images from different angles with complete pars tensa and pars flaccida were adopted from each ear with at least $500 \times 500$ pixels. White light, eardrum size more than $50 \%$ in the otoscopic images and light reflection without overexposure and underexposure were optimal. Otoscopic images with tympanostomy tube, secretion and earwax more than $25 \%$ of tympanic membranes were excluded in this study.

\section{Clinical labelling of otoscopic images}

Only a few parts of otoscopic images have been recorded the presence of attic retraction pocket and atelectasis in the electronic medical record systems. To achieve a consistent ground truth label, we didn't adopt these records as ground truth label. Firstly, JBZ with more than 3 years clinical experience in otology were assigned to address the presence of attic retraction pocket and atelectasis according to the first widespread standard independently ${ }^{17,18}$. Because attic retraction pocket and atelectasis may present in the same otoscopic images, these two lesions were labeled separately. All otoscopic images were labeled the presence of attic retraction pocket and atelectasis without region annotation. Then, two otologists with more than 10 years clinical experience in otology were assigned to review labels independently, and any discrepancy will be discussed with another otologist with more than 20 years clinical experience in otology until consensus was reached. As in actual clinical practice, the prevalence of different stage of attic retraction pocket and atelectasis was heavily skewed in our dataset, stage III and IV attic retraction pocket and atelectasis with less than $5 \%$. To ensure that there was sufficient data to develop and assess the performance of this model, we only address the presence of attic retraction pocket and atelectasis without stage classification. Other clinical demographic data wasn't used to develop deep learning model, such as acoustic test results, age and gender.

\section{Deep learning model development}

3 -fold random cross validation was adopted to divided dataset into training set and validation set. The output of this model was a standard two-class task for determining whether the input otoscopic image contained attic retraction pocket or atelectasis. We used a CNN model pretrained on the ImageNet dataset (http://www.image-net.org), then otoscopic images of this dataset were used to fine-tune the hyperparameters of the pretrained CNN model. During the process of training, online data was used for data expansion, including random vertical and horizontal flip, and constant aspect ratio scaling. Considering our previous experience, Google Inception-V3 were suitable for developing deep learning model based on otoscopic images. So, Google Inception-V3 CNN model was adopted as the backbone network and trained, tuned and evaluated ${ }^{19}$. All the otoscopic images were turned into $299 \times 299$ pixels as input data. CNN model consisted of a convolutional neural network to implicitly recognize characteristics of attic retraction pocket and atelectasis from otoscopic images.

To evaluate the CNN model performance in clinical practice, we compared the predicted diagnosis with the labeled standard diagnosis using the 3-fold average classification accuracy, sensitivity, specificity of the model 
(normal pars flaccida vs attic retraction pocket, normal pars tensor vs atelectasis). We also used receiver operating characteristic (ROC) curve and corresponding area under ROC curve to show the diagnostic ability of the deep learning model in identifying the presence of attic retraction pocket and atelectasis.

\section{Class Activation Mapping}

Class Activation Mapping (CAM) was employed to visualize the discriminative region in the otoscopic images. CAM used different colors to show different values of deep learning model ranging from blue (no specific region) to red (most discriminative region). Right identification of lesion region with red color in the otoscopic images are essential for clinician to trust the deep learning model. All experiments were operated with Python 3.6 in Keras using Python programming language. The diagnostic model was developed based on TensorFlow and carried out with 4 Titan XP 256 GB GPU.

\section{RESULTS}

We collected an image dataset consist of 6393 OME otoscopic images, of which 3564 (55.74\%) otoscopic images were assessed for attic retraction pocket, and atelectasis was diagnosed in 2460 (38.48\%) otoscopic images. Each otoscopic images were reviewed by at least 3 expert otologists. We used 3 -fold cross-validation for developing and testing the deep convolutional neural network (DCNN) model to detect OME referable attic retraction pocket and atelectasis.

\section{Deep learning model performance}

The AUC of classifying the normal pars flaccida and attic retraction pocket was 0.89 , and the accuracy, sensitivity and specificity were $89 \%, 0.93$ and 0.62 respectively (Fig 1). The DCNN model achieved an AUC of 0.87 in classifying the normal pars tensa and atelectasis, and the accuracy, sensitivity and specificity were $79 \%, 0.71$ and 0.84 respectively (Fig 2).

\section{Class Activation Map}

The heat map of CAM image was generated using the otoscopic images from the validation set. The CAM showed the deep learning model can identify attic retraction pocket with red color accurately, and deeper or bigger attic retraction pocket hold more values (Fig 3). Partial atelectasis and general atelectasis were identified by the deep learning model, and deeper or bigger atelectasis showed more values with red color (Fig 4).

\section{DISCUSSION}

The diagnosis of attic retraction pocket and atelectasis is based on an otoscopic examination. However, diagnosis of ear diseases with otoscopic images is a hard task for general practitioners, pediatricians and otolaryngologists, with averaged accuracy $39 \%-53 \%, 36 \%-51 \%$ and $61 \%-74 \%$ respectively ${ }^{20-22}$. In this study, we developed and validated a deep learning model to identify attic retraction pocket and atelectasis with multi-centers otoscopic images. Our CNN algorithm acquired an AUC of 0.89 for the identification of attic retraction pocket and 0.87 for atelectasis.

Previous studies established deep learning models for the diagnosis of tympanic retraction and achieved an averaged accuracy ranging from $85.78 \%$ to $88.06 \%{ }^{15,16}$. Shie et al ${ }^{15}$ only obtained 856 otoscopic images from one center encompassing almost all otitis media categories. Cha et $\mathrm{al}^{16}$ included 1222 otoscopic images with tympanic retraction and they merged atelectasis and attic retraction pocket into a class. Our study included 6393 OME otoscopic images, of which $55.74 \%$ were identified with attic retraction pocket and $38.48 \%$ were identified with atelectasis. Considering that the attic retraction pocket was limited to the pars flaccida of tympanic membrane, the atelectasis is in the pars tensa of tympanic membrane. During the progress of disease, attic retraction pocket is more likely to progress to cholesteatoma, and atelectasis is likely to evolve to ossicular erosions ${ }^{10}$. Therefore, we labeled and divided the retraction pockets into atelectasis and attic retraction pocket. Compared with previous models, we targeted the attic retraction pocket and atelectasis separately, to our current knowledge, this image classification system was the first to diagnose two types of tympanic membrane lesions. 
Our results showed different region (pars tensa and pars flaccida) retraction on the tympanic membranes with different accuracy. It is reasonable for clinical experience that attic retraction pocket is easier to identify than atelectasis, because normal tympanic membrane shows a mild retraction in pars tensa without retraction in pars flaccida. The reason may be that in non-severe cases, the attic retraction pocket may be subtle, and clinicians may find it difficult to determine whether this is a normal or a grade I attic retraction pocket based on Tos and Sade classification systems ${ }^{17,18}$. On the other hand, cases with severe attic retraction pockets and atelectasis often exposed the ossicles inside the tympanic membrane, and sometimes it is difficult to distinguish between perforation and severe atelectasis.

In order to show the discriminative region of deep learning, CAM highlighted the important area with red color ${ }^{17}$, especially large and deep retraction pockets and atelectasis, which was consistent with otologists. Moreover, our image datasets were representative which were collected from three hospitals with different type of otoscopes and image record systems. Many parameters of otoscopes and systems differs in different hospitals, such as the white balance was not equal in different hospital, even in the same hospital because of different preference of practitioners.

During the procedure of follow up, if attic retraction pocket and atelectasis is suggested, observation should be stop and it's better to triage the patients to otologists. On the other hand, in the clinical practice, this model could be useful for generating diagnosis of attic retraction pocket and atelectasis, which could be assistant for otologists. For young otologists and non-otologists, this model could be used as a study platform to learn attic retraction pocket and atelectasis.

Limitation : Some limitations did exist in our study. Although this CNN algorithm could identify mild and severe attic retraction pocket and atelectasis. However, without enough images of severe attic retraction pocket and atelectasis, it is not easy to develop and validate a deep learning model to identify different level of attic retraction pocket and atelectasis. Moreover, accurate segment labeling techniques may be helpful for improving the accuracy of model. We developed the deep learning model with weak supervision, and further detailed annotation before model development are suggested. Thirdly, non-medical history and hearing information were provided to the deep learning model and otolaryngologists, which may affect the accuracy of diagnosis. The doctor can be greatly improved the accuracy of diagnosis by adding disease history.

\section{CONCLUSION}

In summary, we developed a deep learning model which could be useful in helping junior otolaryngologists and non-otolaryngologists to diagnose attic retraction pocket and atelectasis.

CONFLICT OF INTEREST The authors have no potential conflicts of interest with respect to the research, authorship and/ or publication of this article.

CONSENT TO PARTICIPATE The participants signed formal consent to share in this study.

CONSENT FOR PUBLICATION Additional informed consent was obtained from all participants to share and publish their data in this article.

DATA AVAILABILITY STATEMENT The datasets used and analysed during the current study are available from the corresponding author on reasonable request.

\section{REFERENCES}

1. Ungkanont K, Charuluxananan S, Komoltri C. Association of otoscopic findings and hearing level in pediatric patients with otitis media with effusion. Int J Pediatr Otorhinolaryngol. 2010;74(9):1063-1066.

2. Pless RSIBPIB. Hearing Thresholds and Tympanic Membrane Sequelae in Children Managed Medically or Surgically for Otitis Media With Effusion.Arch Pediatr Adolesc Med. 2005;159:1151-1156.

3. De Beer BA, Schilder AG, Zielhuis GA, Graamans K. Natural course of tympanic membrane pathology related to otitis media and ventilation tubes between ages 8 and 18 years. Otol Neurotol.2005;26(5):1016-1021. 
4. Kim GW, Jung HK, Sung JM, Kim JS, Kim CW. A tiny retraction of the pars flaccida may conceal an attic cholesteatoma. Eur Arch Otorhinolaryngol. 2020;277(3):735-741.

5. Rosenfeld RM, Shin JJ, Schwartz SR, et al. Clinical Practice Guideline: Otitis Media with Effusion Executive Summary (Update).Otolaryngol Head Neck Surg. 2016;154(2):201-214.

6. Alzahrani M, Saliba I. Tympanic membrane retraction pocket staging: is it worthwhile? Eur Arch Otorhinolaryngol.2014;271(6):1361-1368.

7. Saunders JE. Does early surgical intervention of middle ear atelectasis improve long-term results and prevent cholesteatoma? Arch Otolaryngol Head Neck Surg. 2008;134(10):1040-1044.

8. Spielmann P, Mills R. Surgical management of retraction pockets of the pars tensa with cartilage and perichondrial grafts. J Laryngol Otol. 2006;120(9):725-729.

9. Garcia de Hombre AM. [Bibliographic revision of retraction pockets handling in relation to surgical treatment]. An Otorrinolaringol Ibero Am. 2005;32(3):221-228.

10. Alper C, Olszewska E. Assessment and management of retraction pockets. Otolaryngol Pol. 2017;71(1):121.

11. Samra S, Wu A, Redleaf M. Interactive iPhone/iPad App for Increased Tympanic Membrane Familiarity. Ann Otol Rhinol Laryngol.2016;125(12):997-1000.

12. Ni G, Curtis S, Kaplon A, et al. Development of video otoscopy quiz using a smartphone adaptable otoscope. J Otol. 2021;16(2):80-84.

13. Myburgh HC, van Zijl WH, Swanepoel D, Hellstrom S, Laurent C. Otitis Media Diagnosis for Developing Countries Using Tympanic Membrane Image-Analysis. EBioMedicine. 2016;5:156-160.

14. Wu Z, Lin Z, Li L, et al. Deep Learning for Classification of Pediatric Otitis Media. Laryngoscope. 2021;131(7):E2344-E2351.

15. Shie CK, Chang HT, Fan FC, Chen CJ, Fang TY, Wang PC. A hybrid feature-based segmentation and classification system for the computer aided self-diagnosis of otitis media. Annu Int Conf IEEE Eng Med Biol Soc. 2014;2014:4655-4658.

16. Cha D, Pae C, Seong SB, Choi JY, Park HJ. Automated diagnosis of ear disease using ensemble deep learning with a big otoendoscopy image database. EBioMedicine. 2019;45:606-614.

17. Sade J, Berco E. Atelectasis and secretory otitis media. Ann Otol Rhinol Laryngol. 1976;85(2 Suppl 25 Pt 2):66-72.

18. Tos M, Stangerup SE, Larsen P. Dynamics of eardrum changes following secretory otitis. A prospective study. Arch Otolaryngol Head Neck Surg. 1987;113(4):380-385.

19. Szegedy C, Vanhoucke V, Ioffe S, Shlens J, Wojna Z. Rethinking the Inception Architecture for Computer Vision. Proc Cvpr Ieee.2016:2818-2826.

20. Pichichero ME, Poole MD. Assessing diagnostic accuracy and tympanocentesis skills in the management of otitis media. Arch Pediatr Adolesc Med. 2001;155(10):1137-1142.

21. Pichichero ME, Poole MD. Comparison of performance by otolaryngologists, pediatricians, and general practioners on an otoendoscopic diagnostic video examination. Int J Pediatr Otorhinolaryngol. 2005;69(3):361-366.

22. Buchanan CM, Pothier DD. Recognition of paediatric otopathology by General Practitioners. Int $J$ Pediatr Otorhinolaryngol.2008;72(5):669-673.

Figure legends 
Figure 1 Receiver-Operator Characteristic (ROC) curves and corresponding area under the ROC curve (AUC) of deep learning model for detection of attic retraction pocket.

Figure 2 Class activation maps of deep learning model for detection attic retraction pocket.

Figure legend: Class activation maps of identification of attic retraction pockets. Red color part represents discriminative region in the otoscopic images, while blue color part represents no-specific region in the otoscopic images. (a) normal pars flaccida. (b) - (d) attic retraction pocket.

Figure 3 Receiver-Operator Characteristic (ROC) curves and corresponding area under the ROC curve (AUC) of deep learning model for detection of atelectasis.

Figure 4 Class activation maps of deep learning model for detection of atelectasis.

Figure legend: Class activation maps of deep learning model for detection of atelectasis. Red color part represents discriminative region in the otoscopic images, while blue color part represents no-specific region in the otoscopic images. (A) normal pars tensa. (B) - (D) atelectasis and attic retraction pocket.

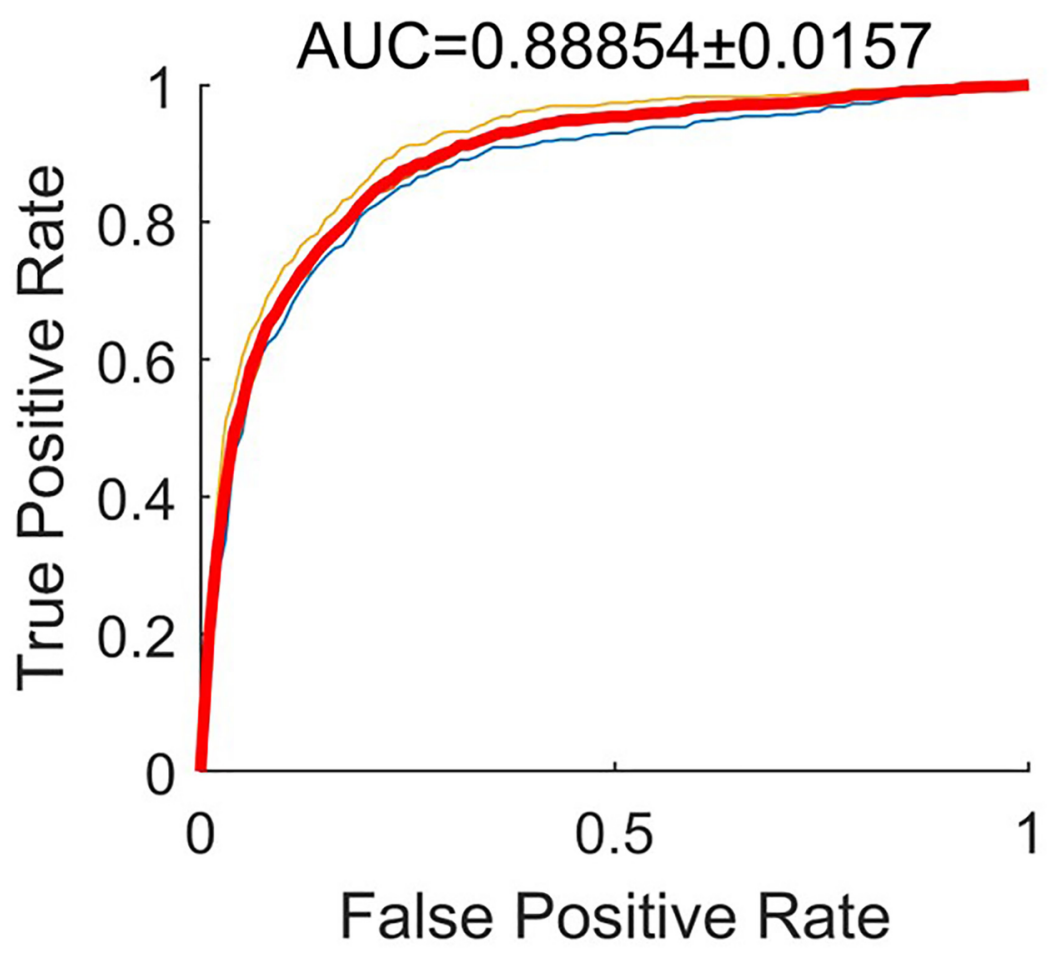



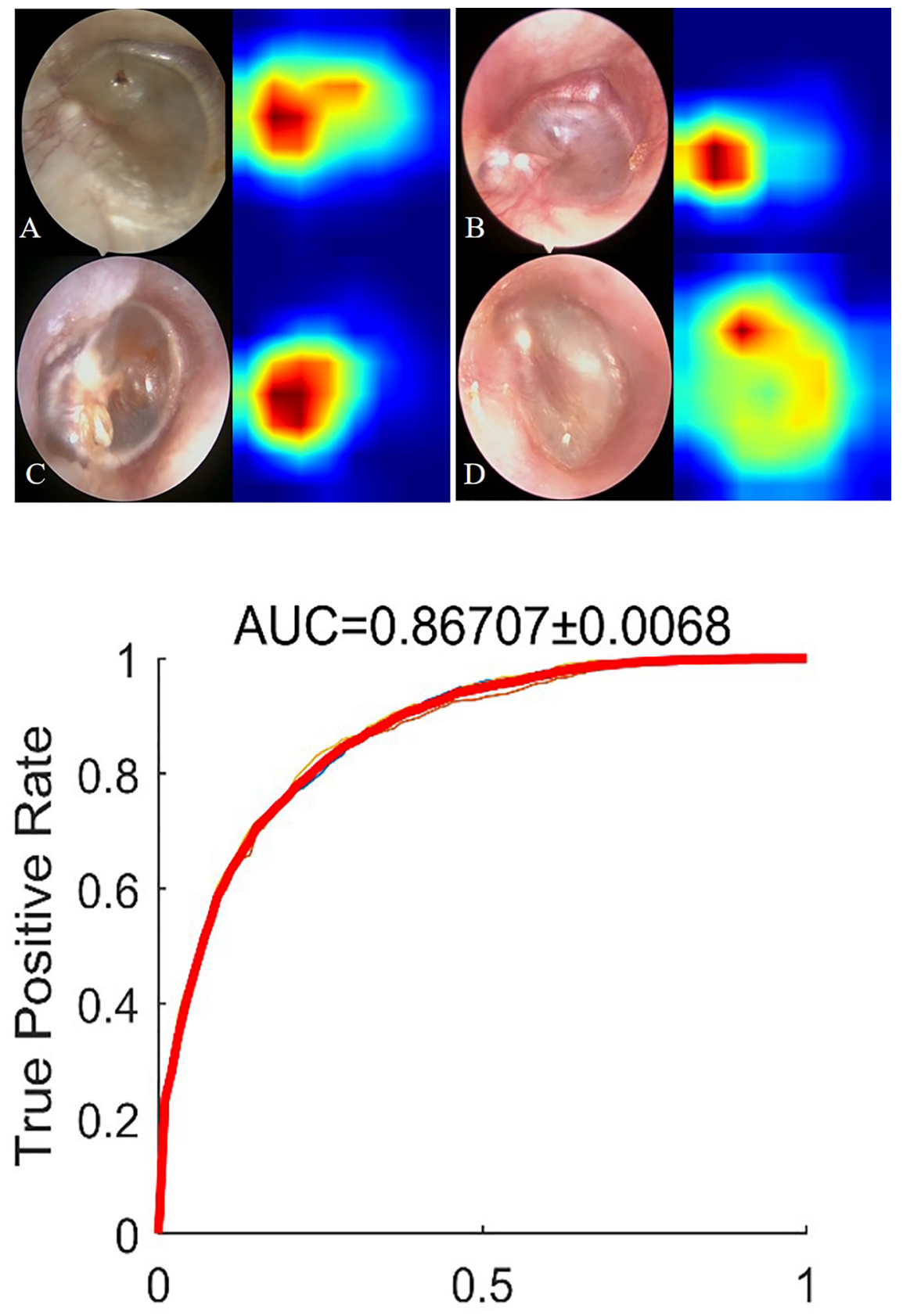

False Positive Rate 


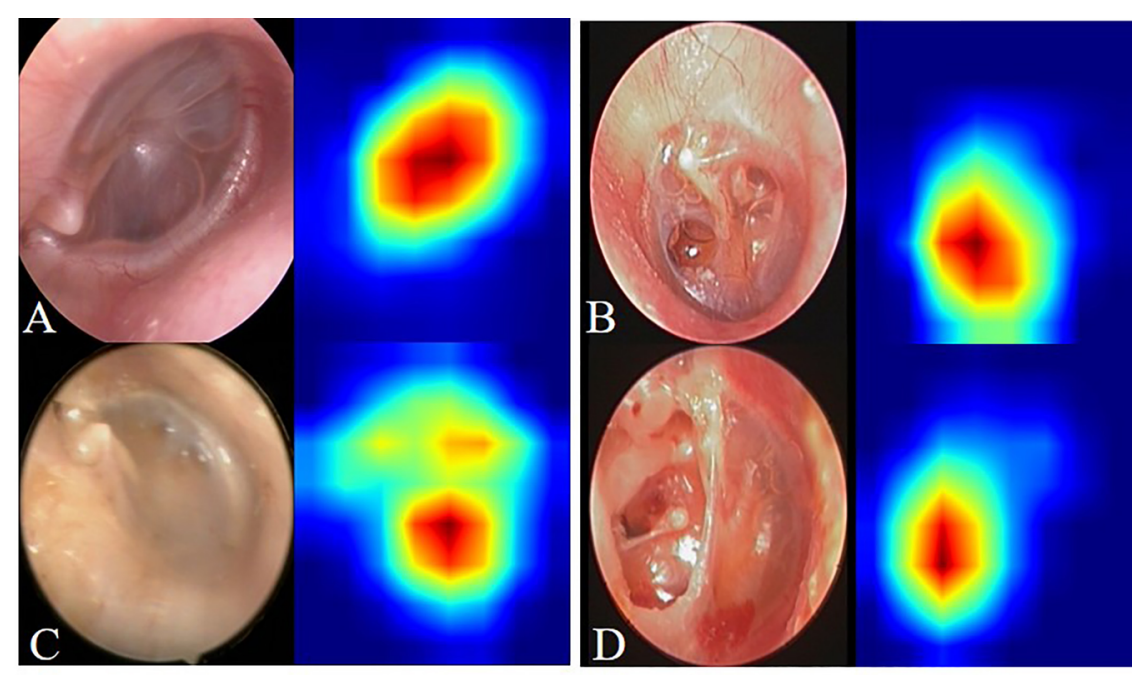

